\title{
acBF: A High Accuracy Membership Filter using $\mathrm{rDBF}$
}

\author{
$1^{\text {st }}$ Ripon Patgiri \\ $2^{\text {nd }}$ Sabuzima Nayak \\ $3^{\text {rd }}$ Samir Kumar Borgohain \\ Dept. of Computer Science \& Engg. \\ Dept. of Computer Science \& Engg. \\ Dept. of Computer Science \& Engg. \\ National Institute of Technology Silchar National Institute of Technology Silchar National Institute of Technology Silchar \\ Assam-788010, India \\ Assam-788010, India \\ email address \\ sabuzimanayak@gmail.com \\ Assam-788010, India
samir@nits.ac.in
}

\begin{abstract}
Bloom Filter is a data structure for membership query which is deployed in diverse research domains to boost up system's performance and to lower on-chip memory consumption. However, there are still lacking of a high accuracy Bloom Filter without compromising the performance and memory space. Moreover, the scalability causes more memory consumption as well as time complexity. Therefore, in this paper, we present a novel Bloom Filter, called accurate Bloom Filter (acBF), which features: a) an impressive guaranteed accuracy of $99.98 \%$, b) a maximum false positive probability of 0.00015 , c) lower collision probability, d) free from false negative, e) optimal insertion and membership query cost, and g) $\leq 8$-bits of memory consumption per item. acBF deploys eight multidimensional Bloom Filter. These multidimensional Bloom Filters eliminate the false positives at eight stages without sacrificing the system performance. We have conducted rigorous experiments to validate the accuracy of acBF which is unprecedentedly high. Also, acBF is compared with Scalable Bloom Filter (SBF) and Cuckoo Filter (CF). Experiments show acBF outperforms SBF and CF in terms of accuracy, and scalability. Moreover, performance of acBF outperforms CF in lookup operation. But, CF outperforms acBF in insertion. However, accuracy of acBF is incomparable with both SBF and CF.

Index Terms-Bloom Filter, Membership Filter, Multidimensional Bloom Filter, Multilevel Bloom Filter, Accuracy, Data Structure, Algorithm, Named Data Networking, Information Centric Network
\end{abstract}

\section{INTRODUCTION}

Bloom Filter [1] is a very famous hash algorithm that boosts up system performance. The popularity of Bloom Filter is increasing day-by-day and it is applied numerous research fields. However, Bloom Filter can not stand itself, but it works as an enhancer of a system. For instance, BigTable [2] uses Bloom Filter to enhance the lookup performance. Moreover, Bloom Filter uses a tiny amount of memory space to keep information of a very large set of data. Bloom Filter consumes least amount memory as compared to the conventional hash algorithm. Bloom Filter is extensively experimented probabilistic data structure since its inception. Bloom Filter is an approximation membership filter with some errors [1]. But, it is deployed in various domains, namely, Big Data [2], Network Security [3], [4], Network Traffic, IoT [5], Biometrics [6], Cloud Computing [7], and Bioinformatics [8], [9]. BigTable

Authors would like to acknowledge TEQIP-III, National Institute of Technology Silchar for funding this work. reduces unnecessary HDD access by deploying Bloom Filter [2]. Moreover, Bloom Filters are deployed in diverse network applications, namely, Network Security [4], [10]-[12], Web Cache, Routing Algorithms [13], [14], Content-centric Network [15]-[19], Named Data Networking [20]-[23], Peer to Peer networking, IoT [5] and WSN [24]. Besides, there are an abundant of network devices that depends on Bloom Filter. Thus, there is an immense necessity of highly accurate Bloom Filter in Computer Networking as well as other domain. Because, Bloom Filter fosters the system performance and reduce the main memory consumption. Moreover, Bloom Filter helps in the development of an unprecedented idea. Bloom Filters consumes an impressively very low amount of memory to store information of very large data. However, false positive and false negative are crucial issues in Bloom Filter.

\section{A. Terminology}

Let, $S$ be a set, $K=K_{1}, K_{2}, K_{3}, \ldots, K_{n}$ be the elements where $K \in S$ and $n$ is the total number of elements [25]. Let, $K_{i}$ be a random query element where $i=1,2,3,4, \ldots$ and $B$ be the Bloom Filter, and therefore, false positive, true positive, false negative, and true positive are defined as follows-

- False Positive: If $K_{i} \in B$ and $K_{i} \notin S$, then the result of Bloom Filter is a false positive.

- True Positive: If $K_{i} \in B$ and $K_{i} \in S$, then the result of Bloom Filter is a true positive.

- False Negative: If $K_{i} \notin B$ and $K_{i} \in S$, then the result Bloom Filter is a false negative.

- Ture Negative: If $K_{i} \notin B$ and $K_{i} \notin S$, then the result of Bloom Filter is a true negative.

\section{B. Motivation}

Bloom Filter is a probabilistic data structure to implement approximate membership query. The Bloom Filter returns either true or false. The "true" result can be classified into either true positive or false positive. Similarly, "negative" result of Bloom Filter is also classified into either true negative or false negative. The research community is trying to eradicate false positive and false negative completely from Bloom Filter, but unable to do so. Accuracy of Bloom Filter depends on the false positive probability (FPP) of the filter. There are many variants of Bloom Filter which are introduced to reduce the 
false positive and false negative, namely, Blocked Bloom Filter [26], Cuckoo Bloom Filter [27], d-Left CBF (dlCBF) [28], Quotient Filter (QF) [29], Scalable Bloom Filter (SBF) [30], Sliding Bloom Filter [31], TinySet [32], Ternary Bloom Filter (TBF) [33], Bloofi [34], BloomFlow [35], Difference Bloom Filter (DBF) [36], and Dynamic Reordering Bloom Filter [37]. The common objectives of these Bloom Filters are

- To reduce false positive and false negative.

- To optimize space consumption.

- To boost up the system performance.

- To make highly adaptable and scalable.

Filtering Millions of keys are a burning issue of modern Bloom Filter because of false positive. Furthermore, Filtering Billion of keys is an unimaginable high input. Large scale membership filter is to sacrifice either performance or memory. Besides, large scale membership filter exhibits more false positive. Forest-Structured Bloom Filter [38], BloomStore [39], and BloomFlash [40] are able to filter at a scale of Billions with the help of Flash memory. However, lookup and insertion cost is higher. In addition, reduction of false positive is an important issue in Bloom Filter. Most of the Bloom Filter suffers from high false positive. On the contrary, Scalable Bloom Filter [30] is able to filter millions of keys. Similarly, Cuckoo Filter [27] too.

\section{Objectives of acBF}

The key objectives of acBF are outlined as follows-

- To reduce false positive,

- To increase accuracy,

- To reduce memory consumption while maintaining high accuracy,

- To increase performance while maintaining a low false positive probability.

- And, to get independent from number of hash function $k$,

\section{Contribution}

In this paper, we present a novel high accuracy Bloom Filter using rDBF [41], called acBF, which features as follows-

- acBF offers a high accuracy Bloom Filter and its accuracy is as high as $99.98 \%$.

- The false positive probability of acBF is as low as 0.00015 .

- acBF also offers an impressive collision probability which is $\ll \frac{1}{2}$,

- acBF eliminates false positive through an eight stage Bloom Filter without sacrificing the overall performance.

- acBF is free from false negative,

- Both, the time complexity of insertion and membership testing of acBF are $O(1)$, and

- acBF guarantees memory consumption $\leq 8$ - bits per input item irrespective of input item size.

The rDBF filters are very fast [41] and these filters use a single bit to keep information of an input item. Therefore, acBF does not suffer from performance issue.

\section{E. Organization}

acBF deploys four variants of rDBF [41]. These variants are exposed in Section II. Section II establishes our proposed system. Section III proves the impact of our propose system through a rigorous experimentation. Section IV performs various theoretical analysis on acBF. Moreover, Section V discusses on pros and cons of acBF. Finally, the paper is concluded in Section VI.

\section{Proposed System}

Our proposed system deploys four variants of rDBF [41], and an eight stage filter removes false positive. The proposed system aims to remove false positive and increase accuracy. An item is inserted into the eight Bloom Filter without maintaining an order. On the contrary, our proposed system maintains serial order of membership query to ensure the true positiveness of a key. The proposed system uses Two Dimensional Bloom Filter (2DBF), Three Dimensional Bloom Filter (3DBF), Four Dimensional Bloom Filter (4DBF), and Five Dimensional Bloom Filter (5DBF). An item is inserted into 2DBF at Stage-I and reversal of the key item is inserted into $2 \mathrm{DBF}^{\prime}$ at Stage-II. Similarly, the same input item is inserted into 3DBF at StageIII and the reversal of the key item is inserted into $3 \mathrm{DBF}^{\prime}$ at Stage-VI, and so on. Therefore, there are eight filters and eight stages of the filter in acBF.

\section{A. Two Dimensional Bloom Filter}

1) Insertion and querying at Stage-I: At the very beginning, acBF deploys Two Dimensional Bloom Filter (2DBF) [41]. 2DBF [41] uses three modulus operations to place an item in a particular bit position instead of placing an item in various locations in the Bloom Filter. Thus, it improves the space performance significantly.

Let us assume, $B_{M, N}$ be the 2-dimensional array to implement Bloom Filter which is initialized by zero. $M$ and $N$ be the dimensions of the filter. The necessary condition for $M$ and $N$ are prime numbers. It requires three parameters to set a bit in $B_{M, N}$, namely, $i, j$, and pos where pos is the bit position of a particular cell, say, $B_{i, j}$. The cell size of $B_{i, j}$ depends on the memory occupied by the filter for each cell, termed as $\beta$, for example, 64 - bits. Now, 2DBF sets a bit in $B_{i, j}$ by invoking Equation (1) as follows-

$$
B_{i, j} \leftarrow B_{i, j} \text { OR }(1<<p o s)
$$

where $O R$ is a bitwise operator. The Equation (1) is used to set a bit. Let, $K$ be an item to insert in 2DBF. Now, the Murmur hash function $\mathcal{H}(K)$ returns a value and assigned the returned value to $h$ by $h \leftarrow \mathcal{H}(K)$. Instead of placing $K$ in various locations, $2 \mathrm{DBF}$ performs three modulus operations and place it in a single bit position. To place the item $K$, 2DBF calculates following parameters as follows- $i \leftarrow h \% M$, $j \leftarrow h \% N$, and pos $\leftarrow h \% \beta$, where $\%$ is a modulus operator and $\beta$ is the bit size per cell of the Bloom array. Thus, the item $K$ is inserted using the Equation (1). 
Similarly, Equation (2) is invoked to test whether a particular bit is set or not.

$$
\text { Flag }_{1} \leftarrow\left(B_{i, j} \oplus(1<<\text { pos })\right) A N D(1<<\text { pos })
$$

where $A N D$ is a bitwise operator. If $F l a g_{1}$ and $B_{i, j}$ are zero, then the particular bit is set to zero, one otherwise. In addition, membership of $K$ is tested using the Equation (2) same $i$, $j$, and pos. So, an item requires a single bit in $2 \mathrm{DBF}$ and reduces the memory consumption significantly as well as the false positive probability.

2) Insertion and querying at Stage-II: To remove the false positive of $2 \mathrm{DBF}$, acBF deploys another $2 \mathrm{DBF}$ which is termed as $2 \mathrm{DBF}^{\prime}$. The input item $K$ is reversed and inserted into $2 \mathrm{DBF}^{\prime}$. Let $K^{\prime}$ be the reversal of input item $K$. Hence, $K \neq$ $K^{\prime}$, since $K^{\prime}$ is reversal of $K$, except palindromic input. The input item $K^{\prime}$ is inserted to the $2 \mathrm{DBF}^{\prime}$ using Equation (3).

$$
B_{i^{\prime}, j^{\prime}}^{\prime} \leftarrow B_{i^{\prime}, j^{\prime}}^{\prime} \text { OR }\left(1<<\operatorname{pos}^{\prime}\right)
$$

Let, hash function $h^{\prime}=\mathcal{H}\left(K^{\prime}\right)$ returns a value. The $K^{\prime}$ is the reversal of input item $K$. Thus, $i^{\prime} \leftarrow h^{\prime} \% M, j^{\prime} \leftarrow h^{\prime} \% N$, and pos $^{\prime} \leftarrow h^{\prime} \% \beta$. Thus, Equation (3) is invoked to insert input item $K^{\prime}$ into $2 \mathrm{DBF}^{\prime}$.

In a membership testing of a key $K^{\prime}$, following Equation (4) must satisfy-

$$
\begin{aligned}
& \text { Flag }_{2} \leftarrow \text { Flag }_{1} \text { AND }\left(\left(B_{i^{\prime}, j^{\prime}}^{\prime} \oplus\right.\right. \\
& \left.\left.\left(1<<\operatorname{pos}^{\prime}\right)\right) A N D\left(1<<\operatorname{pos}^{\prime}\right)\right)
\end{aligned}
$$

Equation (4) reduces false positive, but not free from false positive.

\section{B. Three Dimensional Bloom Filter}

1) Insertion and querying at Stage-III: acBF deploys Three Dimensional Bloom Filter (3DBF) [41] at Stage-III. Let us assume, $B_{P, Q, R}$ be the 3-dimensional array to implement Bloom Filter which is initialized by zero. The $P, Q$, and $R$ are the dimensions of the filter and these are prime numbers. Similar to $2 \mathrm{DBF}$, acBF set a bit in $B_{i, j, k}$ by Equation (5) as follows-

$$
B_{i, j, k} \leftarrow B_{i, j, k} \text { OR }(1<<\text { pos })
$$

where $i, j$, and $k$ are calculated using $i \leftarrow h \% P, j \leftarrow h \% Q$, $k \leftarrow h \% R$, and $p o s=h \% \beta$.

Similarly, Equation (6) is invoked at Stage-III to test whether a particular bit is set or not.

$$
\begin{array}{r}
\text { Flag }_{3} \leftarrow \text { Flag }_{2} \text { AND }\left(\left(B_{i, j, k} \oplus\right.\right. \\
(1<<\text { pos })) A N D(1<<\text { pos }))
\end{array}
$$

If $\mathrm{Flag}_{3}$ are zero, then the particular bit is set to zero, one otherwise.

2) Insertion and querying at Stage-IV: To remove false positive from $3 \mathrm{DBF}$, acBF apply another $3 \mathrm{DBF}$, called $3 \mathrm{DBF}^{\prime}$. The input item $K^{\prime}$ is inserted to the $3 \mathrm{DBF}^{\prime}$ using Equation (7).

$$
B_{i^{\prime}, j^{\prime}, k^{\prime}}^{\prime} \leftarrow B_{i^{\prime}, j^{\prime}, k^{\prime}}^{\prime} \text { OR }(1<<\text { pos })
$$

where $i^{\prime}, j^{\prime}$, and $k^{\prime}$ are calculated using $i^{\prime} \leftarrow h^{\prime} \% P, j^{\prime} \leftarrow$ $h^{\prime} \% Q, k^{\prime} \leftarrow h^{\prime} \% R$, and $\operatorname{pos}^{\prime}=h^{\prime} \% \beta$.
In a membership testing of a key $K^{\prime}$, following Equation (8) must satisfy at Stage-IV:

$$
\begin{array}{r}
\text { Flag }_{4} \leftarrow \text { Flag }_{3} \text { AND }\left(\left(B_{i^{\prime}, j^{\prime}, k^{\prime}}^{\prime} \oplus\right.\right. \\
\left.\left.\left(1<<\operatorname{pos}^{\prime}\right)\right) A N D\left(1<<\operatorname{pos}^{\prime}\right)\right)
\end{array}
$$

\section{Four Dimensional Bloom Filter}

1) Insertion and querying at Stage-V: acBF also applies Four Dimensional Bloom Filter (4DBF) [41]. Let us assume, $B_{w, x, y, z}$ be the 4-dimensional array to implement Bloom Filter which is initialized by zero. The $w, x, y$ and $z$ are the dimensions of the filter. acBF set a bit in $B_{i, j, k, l}$ by Equation (9) as follows-

$$
B_{i, j, k, l} \leftarrow B_{i, j, k, l} \text { OR }(1<<p o s)
$$

where $i, j, k$ and $l$ are calculated using $i \leftarrow h \% W, j \leftarrow$ $h \% X, k \leftarrow h \% Y, l \leftarrow h \% Z$, and pos $=h \% \beta$.

Likewise, Equation (10) is invoked at Stage-V to test whether a particular bit is set or not.

$$
\begin{array}{r}
\text { Flag }_{5} \leftarrow \text { Flag }_{4} \text { AND }\left(\left(B_{i, j, k, l} \oplus\right.\right. \\
(1<<\text { pos })) A N D(1<<\text { pos }))
\end{array}
$$

2) Insertion and querying at Stage-VI: To remove false positive from $4 \mathrm{DBF}$, acBF creates another $4 \mathrm{DBF}$, called $4 \mathrm{DBF}^{\prime}$. The input item $K^{\prime}$ is inserted to the $4 \mathrm{DBF}^{\prime}$ using Equation (11) at Stage-VI.

$$
B_{i^{\prime}, j^{\prime}, k^{\prime}, l^{\prime}}^{\prime} \leftarrow B_{i^{\prime}, j^{\prime}, k^{\prime}, l^{\prime}}^{\prime} O R\left(1<<\operatorname{pos}^{\prime}\right)
$$

where $i^{\prime}, j^{\prime}, \quad k^{\prime}$ and $l^{\prime}$ are calculated using $i^{\prime} \leftarrow h^{\prime} \% W$, $j^{\prime} \leftarrow h^{\prime} \% X, k^{\prime} \leftarrow h^{\prime} \% Y, l^{\prime} \leftarrow h^{\prime} \% Z$, and $\operatorname{pos}^{\prime}=h^{\prime} \% \beta$.

In a membership testing of a key $K^{\prime}$, following Equation (12) must satisfy at Stage-VI-

$$
\begin{array}{r}
\text { Flag }_{6} \leftarrow \text { Flag }_{5} \text { AND }\left(\left(B_{i^{\prime}, j^{\prime}, k^{\prime}, l^{\prime}}^{\prime} \oplus\right.\right. \\
\left.\left.\left(1<<\operatorname{pos}^{\prime}\right)\right) A N D\left(1<<\operatorname{pos}^{\prime}\right)\right)
\end{array}
$$

\section{Five Dimensional Bloom Filter}

1) Insertion and querying at Stage-VII: acBF deploys Five Dimensional Bloom Filter (5DBF) [41] at Stage-VI. Let us assume, $B_{C, D, E, F, G}$ are the 5-dimensional array to implement Bloom Filter which is initialized by zero. The $C, D, E, F$ and $G$ are the dimension of the filter. acBF set a bit in $B_{i, j, k, l, m}$ by Equation (13) as follows:

$$
B_{i, j, k, l, m} \leftarrow B_{i, j, k, l, m} O R(1<<p o s)
$$

where $i, j, k, l$ and $m$ are calculated using $i \leftarrow h \% C, j \leftarrow$ $h \% D, k \leftarrow h \% E, l \leftarrow h \% F, m \leftarrow h \% G$, and $p o s=h \% \beta$.

Similarly, Equation (14) is invoked at Stage-VII to test whether a particular bit is set or not.

$$
\begin{array}{r}
\text { Flag }_{7} \leftarrow \text { Flag }_{6} \text { AND }\left(\left(B_{i, j, k, l, m} \oplus\right.\right. \\
(1<<\text { pos })) A N D(1<<\text { pos }))
\end{array}
$$


2) Insertion and querying at Stage-VIII: At the final stage, acBF create another $5 \mathrm{DBF}$, called $5 \mathrm{DBF}^{\prime}$. The input item $K^{\prime}$ are inserted to the $5 \mathrm{DBF}^{\prime}$ using Equation (15)

$$
B_{i^{\prime}, j^{\prime}, k^{\prime}, l^{\prime}, m^{\prime}}^{\prime} \leftarrow B_{i^{\prime}, j^{\prime}, k^{\prime}, l^{\prime}, m^{\prime}}^{\prime} \text { OR }\left(1<<\text { pos }^{\prime}\right)
$$

where $i^{\prime}, j^{\prime}, k^{\prime}, l^{\prime}$ and $m^{\prime}$ are calculated using $i^{\prime} \leftarrow h^{\prime} \% C$, $j^{\prime} \leftarrow h^{\prime} \% D, k^{\prime} \leftarrow h^{\prime} \% E, l^{\prime} \leftarrow h^{\prime} \% F, m^{\prime} \leftarrow h^{\prime} \% G$, and $\operatorname{pos}^{\prime}=h^{\prime} \% \beta$.

In a membership testing of a key $K$, following Equation (16) must satisfy at the final stage-

$$
\begin{aligned}
\text { Flag }_{8} & \leftarrow \text { Flag }_{7} \text { AND }\left(\left(B_{i^{\prime}, j^{\prime}, k^{\prime}, l^{\prime}, m^{\prime}}^{\prime} \oplus\right.\right. \\
& \left.\left.\left(1<<\operatorname{pos}^{\prime}\right)\right) A N D\left(1<<\text { pos }^{\prime}\right)\right)
\end{aligned}
$$

The Equation (1), (3), (5), (7), (9), (11), (13), and (15) are invoked in insertion of a key. However, it is guaranteed that the Equation (2), (4), (6), (8), (10), (12), (14), and (16) are invoked in true positive. It is not necessary to invoke all Equations in negative results.

\section{E. Insertion of an item}

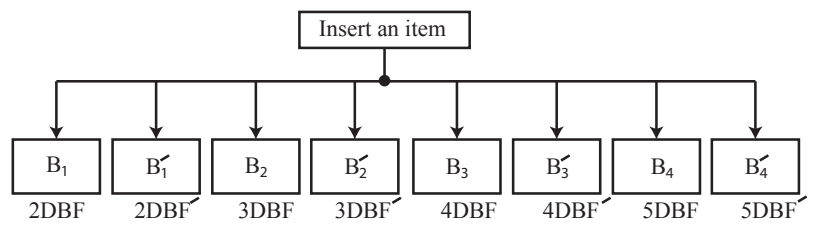

Fig. 1. Insertion of an item into acBF.

acBF deploys 2DBF, 2DBF', 3DBF, 3DBF', 4DBF, 4DBF', $5 \mathrm{DBF}$ and $5 \mathrm{DBF}$ ' for removing false positive. An item is inserted into acBF in any order or in parallel as depicted in Figure 1. Equation (1), Equation (3), Equation (5), Equation (7), Equation (9), Equation (11), Equation (13), and Equation (15) are invoked to insert an item. An item is inserted into eight filters, and each item occupies a single bit in each filter. acBF uses 14 prime numbers for dimensions and each filter maps a key in a different slot.

\section{F. Membership query}

The $2 \mathrm{DBF}$ is faster than 3DBF, 4DBF, and 5DBF. Similarly, $3 \mathrm{DBF}$ is faster than $4 \mathrm{DBF}$ and $5 \mathrm{DBF}$, and so on. On the contrary, 5DBF exhibits lower false positive than 2DBF, 3DBF, and 4DBF. Similarly, 4DBF exhibits lower false positive than 2DBF and 3DBF, and so on. Therefore, at the very beginning, acBF tests the existence of an item in 2DBF as depicted in Figure 2. If $2 \mathrm{DBF}$ returns false, then acBF concludes that there is no such item exist. Otherwise, test the existence of the item in $2 \mathrm{DBF}^{\prime}$. The input item is reversed and input into $2 \mathrm{DBF}^{\prime}$. If $2 \mathrm{DBF}^{\prime}$ returns false, then acBF concludes that there is no such item exist. Otherwise, tests the existence of the item in $3 \mathrm{DBF}$, and so on. It is not necessary to pass through the eight stages on a membership testing in case of negative result. The probability of false positive results returned by $2 \mathrm{DBF}$ is $\frac{1}{3}$. However, there can be a false positive in 2DBF. Hence, the $2 \mathrm{DBF}^{\prime}$ removes the false positive and that probability is $\frac{1}{3}$. The total probability of removing false positive is $\frac{1}{3} \times \frac{1}{3}$ at $2 \mathrm{DBF}^{\prime}$. If $2 \mathrm{DBF}^{\prime}$ is unable to remove false positive, then 3DBF removes false positive, and the probability is $\frac{1}{3^{3}}$ and so on. Therefore, the probability of removing false positive at $5 \mathrm{DBF}^{\prime}$ is

$$
\sum_{i=1}^{8} \frac{1}{3^{i}}=0.00015
$$

Equation (17) derives the probability of false positive at $5 \mathrm{DBF}^{\prime}$. acBF aims to eliminate false positive as early as possible. However, querying a membership on an item must pass all stages of the acBF in case of a positive result. There is no false negative in $2 \mathrm{DBF}, 3 \mathrm{DBF}, 4 \mathrm{DBF}$, and $5 \mathrm{DBF}$ model. If there is a negative outcome from any one of these filters, then it is a true negative.

Let, $\mathcal{R}_{1}, \mathcal{R}_{2}, \mathcal{R}_{3}, \mathcal{R}_{4}, \mathcal{R}_{5}, \mathcal{R}_{6}, \mathcal{R}_{7}$, and $\mathcal{R}_{8}$ are results obtained from 2DBF, 2DBF', 3DBF, 3DBF', 4DBF, 4DBF', $5 \mathrm{DBF}$, and $5 \mathrm{DBF}^{\prime}$ for a membership query of an input item respectively. Thus, the result is concluded by

$$
\mathcal{R}=\prod_{i=1}^{8} \mathcal{R}_{i}
$$

which is represented in the Equation (12). That is, rewriting the Equation (18)

$$
\text { Flag }=\prod_{i=1}^{8} \text { Flag }_{i}
$$

\section{EXPERIMENTAL RESULTS}

\section{A. Data description}

We have generated random numbers of various sizes ranging from $1 \mathrm{M}$ to $64 \mathrm{M}$ to check the false positive, lookup and insertion performance. On the other hands, we have also used Microsoft trace dataset from the IOTTA SNIA repository. We form several sizes of trace data to study the behavior and compare with Scalable Bloom Filter (SBF), Cuckoo Filter (CF) and $\mathrm{acBF}$.

\section{B. Experimental Environment}

TABLE I

EXPERIMENTAL ENVIRONMENTS SETUP

\begin{tabular}{ll}
\hline Name & Description \\
\hline CPU & Intel(R) Core(TM) i7-7700 CPU @ \\
& $3.60 \mathrm{GHz}$ \\
L1 Cache & $32 \mathrm{~K}$ \\
L2 Cache & $256 \mathrm{~K}$ \\
L3 Cache & $8192 \mathrm{~K}$ \\
RAM & $8 \mathrm{~GB}$ \\
HDD & $500 \mathrm{~GB}$ \\
Language & GCC 7.3.0 \\
Operating & Ubuntu 18.04.1 LTS 64-bits \\
System & \\
\hline
\end{tabular}

Table I exposes the experimental environment. We have conducted the experiments on Desktop PC as specified in Table I. 


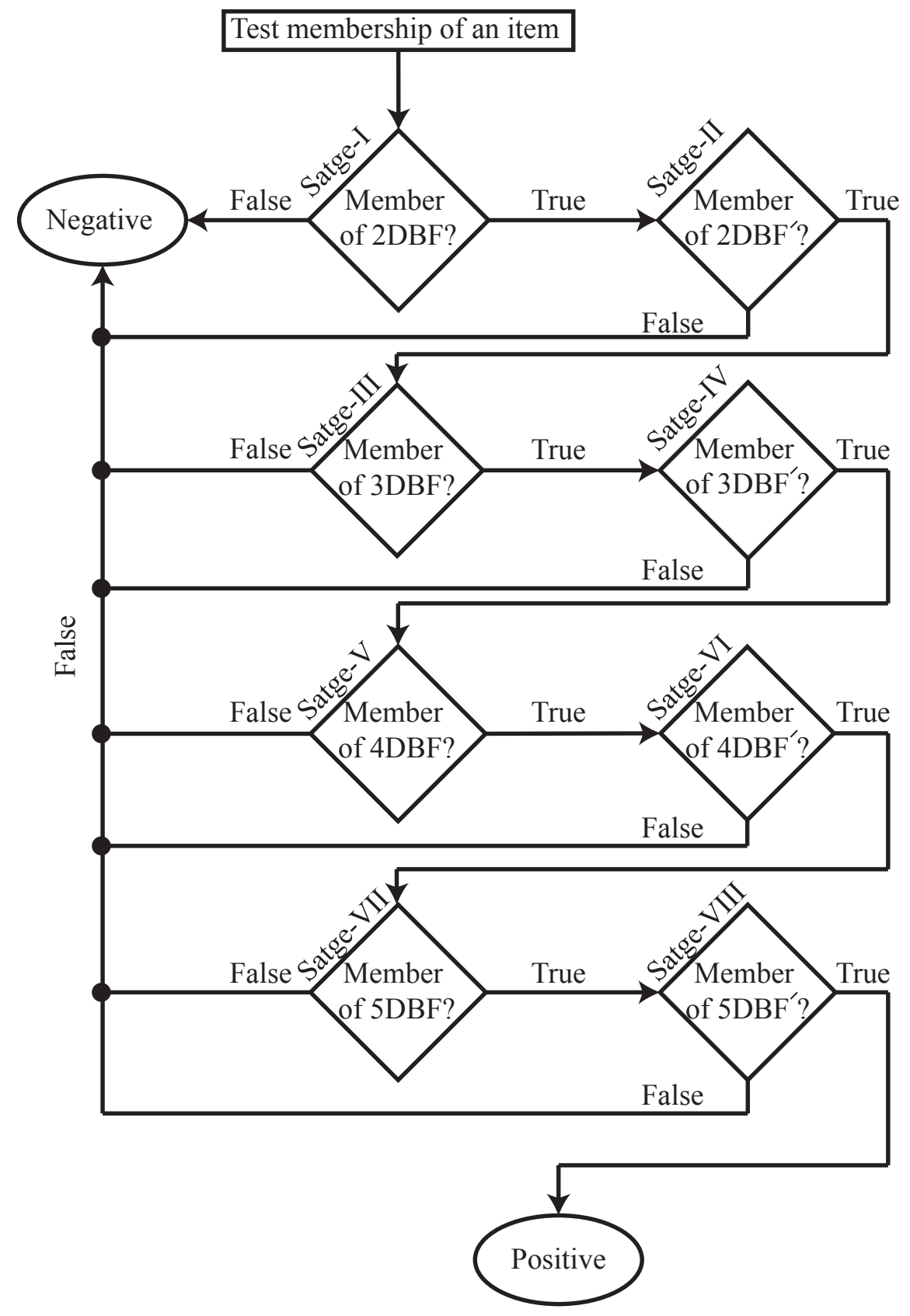

Fig. 2. Membership testing of an item in acBF.

\section{Performance}

acBF offers extremely high performance in the lookup. However, the insertion performance is slower than lookup performance as sketched in Figure 4 and 5 . acBF is an eight stage filter, and thus, acBF provides early detection of nonexistence of an item. On the contrary, an input item is inserted into all eight filters, and thus, it is slower than lookup. Lookup operation does not necessarily pass to all eight stages if the item is not a member of the filter.

Microsoft trace data from IOTTA SNIA [42] is used to benchmark the performance. The datatset is chopped into several small sizes. Figure 4 sketched the performance of acBF, SBF and CF. The lookup performance of acBF is extremely fast. Also, insertion of acBF is faster than $\mathrm{CF}$ and SBF. Most importantly, acBF consumes 3.2MB memory for all operations. It is impressively low memory consumption. On the contrary, SBF and CF consume 19.95MB and $8 \mathrm{MB}$ memory on input size of 62MB (input item 2600643) respectively. Therefore, the space improvement of acBF is $60 \%$ over CF and $83.96 \%$ over SBF. acBF is highly compacted due to rDBF [41], and rDBF does not depend on input item size.

Alternatively, we have generated random numbers ranging 


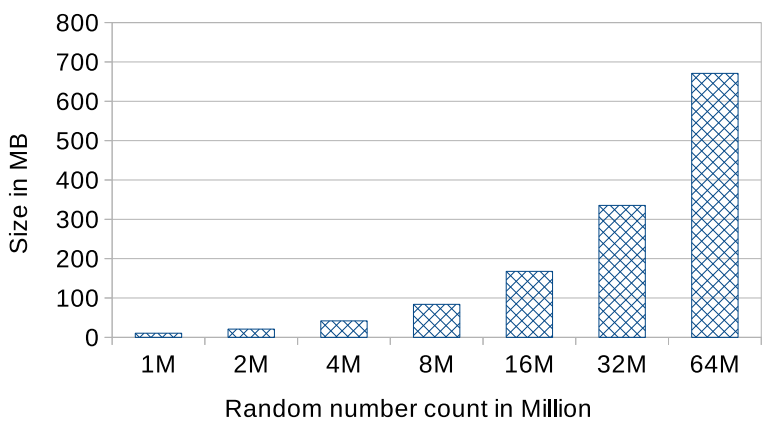

Fig. 3. Random number generations and it's description.

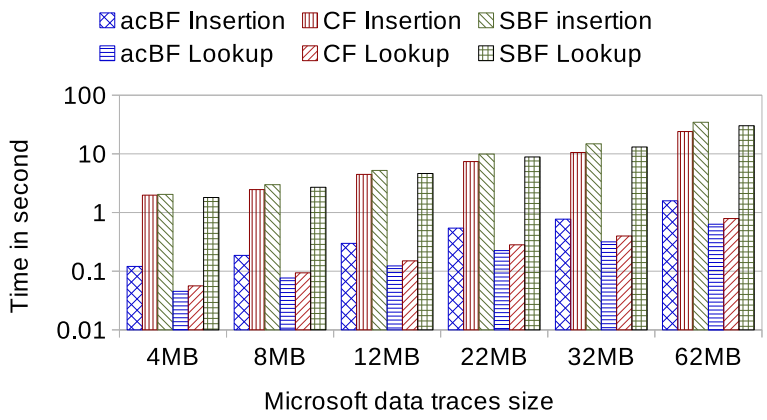

Fig. 4. Time taken to perform insertion and lookup operations on Miscrosoft trace dataset from IOTTA SNIA. Lower is better.

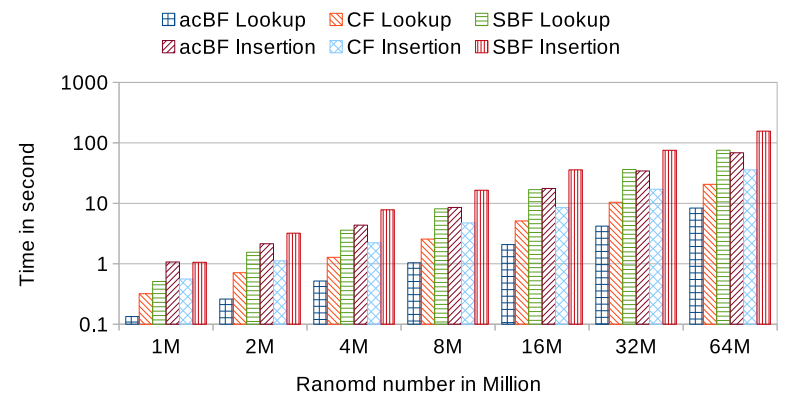

Fig. 5. Time taken to perform insertion and lookup operations on millions of Random numbers. Lower is better.

from $1 \mathrm{M}$ to $1000 \mathrm{M}$. However, SBF starts throwing error from 64M input and CF starts throwing error from $128 \mathrm{M}$ input. These two filters are unable process large number of inputs. acBF outperforms $\mathrm{CF}$, and SBF in lookup operations as depicted in Figure 5. We admit that $\mathrm{CF}$ outperforms acBF and SBF in the insertion of random number. Nevertheless, SBF and CF consume huge on-chip memory on large input size.

Figure 6 extrapolates the throughput on random number input to acBF, $\mathrm{CF}$, and SBF, measured in Million Operations Per Second (MOPS). Lookup throughput of acBF, $\mathrm{CF}$, and SBF are 7.57, 3.12 and 1.41 MOPS on an average respectively. acBF outperforms CF and SBF. Similarly, insertion throughput of acBF, CF, and SBF are 0.93, 1.79 and 0.68 MOPS on an

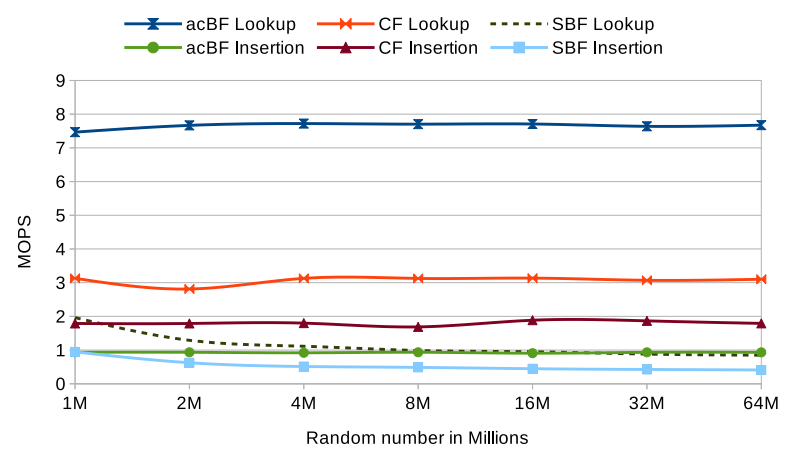

Fig. 6. Throughput of insertions and lookup in Million Operations Per Second (MOPS). Higher is better.

average respectively. $\mathrm{CF}$ defeats acBF and $\mathrm{SBF}$.

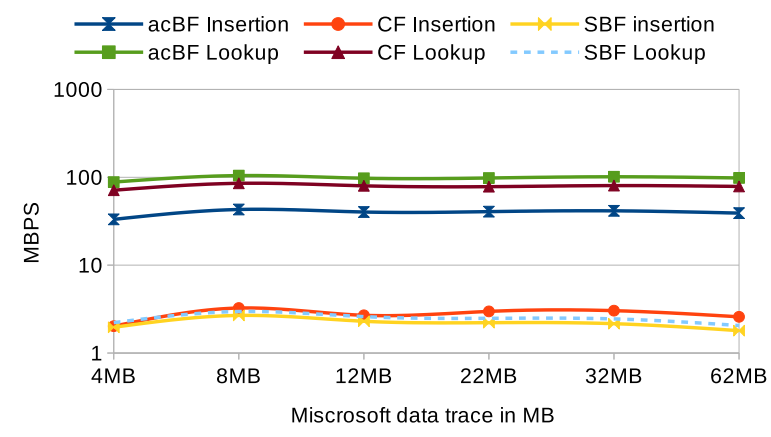

Fig. 7. Throughput of insertions and lookup in Megabytes Operations Per Second (MBPS). Higher is better.

Figure 7 depicts the throughput of insertion and lookup operations of acBF, CF and SBF in Microsoft trace dataset. Insertion throughput of acBF, $\mathrm{CF}$ and $\mathrm{SBF}$ are 36.16, 2.30 and 1.88 MBPS on an average respectively. In this dataset, acBF outperforms CF and SBF in insertion. Similarly, lookup operation of acBF, CF and SBF are 93.29, 74.98 and 2.14 MBPS on an average respectively. Also, acBF outruns $\mathrm{CF}$ and SBF in lookup operation.

\section{False positive}

The key objective of acBF is to achieve high accuracy. acBF emphasizes on high accuracy by eliminating false positive in each stage of the filter. acBF incomparable with $\mathrm{CF}$ and SBF for accuracy. Therefore, Figure 8 demonstrates the total number of false positive found in acBF in different size Microsoft trace dataset while querying by the different size of random numbers ranging from $1 \mathrm{M}$ to $1000 \mathrm{M}$. The false positive rate is impressively very low. In this experiment, false positive occurs from 22MB Microsoft trace dataset and querying by $256 \mathrm{M}$ random numbers. Similarly, a false positive is found at $32 \mathrm{MB}$ and $62 \mathrm{MB}$ Microsoft trace dataset querying by $16 \mathrm{M}$ random numbers. The highest false positive found in $62 \mathrm{MB}$ input and querying $1 \mathrm{~B}$ random number which is accounted as low as 19 as depicted in Figure 8. 


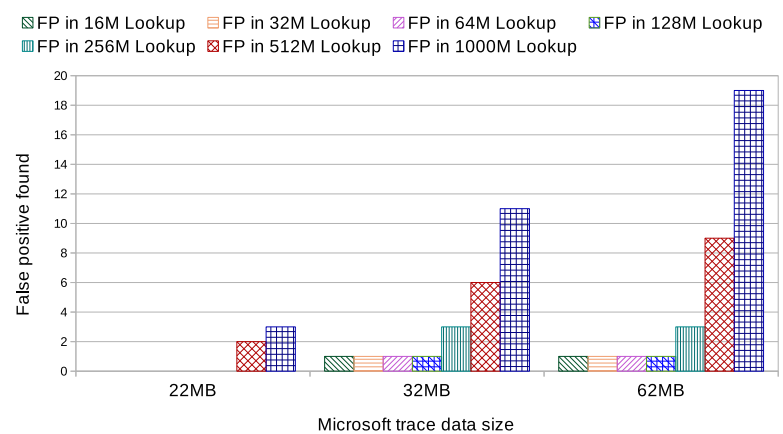

Fig. 8. Number of false positive founds in acBF on Microsoft trace dataset from IOTTA SNIA.

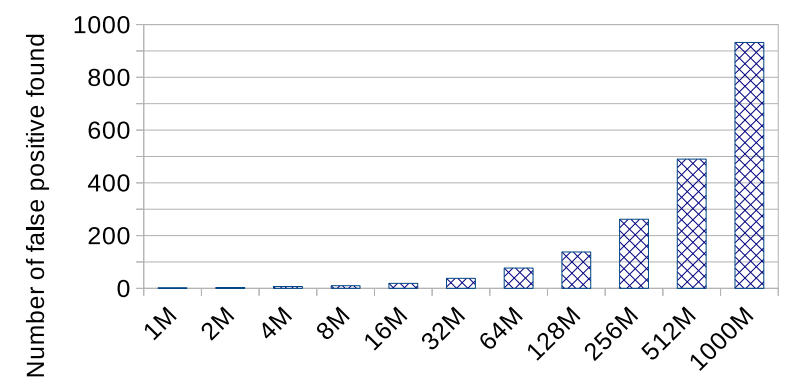

Random number query in Millions

Fig. 9. Number of false positive found in acBF by inputting 43.7 Millions hashtag data from Twitter tweets.

To evaluate the false positive, we inserts 43.7 million hashtag from Twitter [43]. The hashtag data size is 516.3MB. acBF filter size is $40 \mathrm{MB}$ which is comprised of Billions of bits. Then, we have generated random integer with various sizes of data to find false positives. The data size is ranging from 1 million to 1 billion. Figure 9 depicts the total false positive found in the lookup. The false positive in $1 \mathrm{M}$ is as few as 2 and $1 \mathrm{~B}$ is as low as 932 . This is negligible false positive in a very large scale membership filter.

\section{ANALYSIS}

The 2DBF is faster in performance, but higher in false positive rate. Hence, acBF places $2 \mathrm{DBF}$ in the first place. Similarly, 5DBF is higher in accuracy, but slower in performance. Hence, acBF keeps 5DBF in last position to filter. A cell can accommodate many input items, since, an input item occupies a single bit. For example, unsigned long int occupies 8 -bytes. Therefore, the cell can retain information of at most 64 different input items. However, it depends on the prime number $\beta$. If $\beta$ is not a prime number, then the collision probability in a cell is high.

\section{A. False positive}

Let, $m$ bits be the size of Bloom Filter, $n$ be the total number of entries, then the probability of a bit to be ' 0 ' is similar to conventional Bloom Filter, and it is

$$
\left(1-\frac{1}{m}\right)^{n}
$$

Unlike conventional Bloom Filter, number of space occupied by each item is one bit by each filter. Hence, there is no effect of number of hash functions. Therefore, the probability of a bit to be ' 1 ' is

$$
\left(1-\left(1-\frac{1}{m}\right)^{n}\right)
$$

Let $X$ be the random variable of total number of bits filled by ' 1 ' in Bloom Filter is [44]

$$
E[X]=m\left(1-\left(1-\frac{1}{m}\right)^{n}\right)
$$

F. Grandi [44] calculates false positive probability (FPP) through $\gamma$-transformations. Let, $X$ be the random variable representing the total number set bit in the Bloom Filter array, then

$$
E[X]=m\left(1-\left(1-\frac{1}{m}\right)\right)^{h n}
$$

Let us conditioning the random variable $X=x$ to determine the false positive, then

$$
\operatorname{Pr}(F P P \mid X=x)=\left(\frac{x}{m}\right)^{h}
$$

Total probability theorems gives the exact false positive probability as follows

$$
\begin{gathered}
F P P=\sum_{x=0}^{m} \operatorname{Pr}(F P \mid X=x) \operatorname{Pr}(X=x) \\
=\sum_{x=0}^{m}\left(\frac{x}{m}\right)^{h} f(x)
\end{gathered}
$$

where $f(x)$ is a probability density function. F. Grandi [44] performs $\gamma$-transformation to calculate the value of $f(x)$ and the exact false positive probability is

$$
F P P=\sum_{x=0}^{m}\left(\frac{x}{m}\right)^{h}\left(\begin{array}{c}
m \\
x
\end{array}\right) \sum_{j=0}^{x}(-1)^{j}\left(\begin{array}{l}
x \\
j
\end{array}\right)\left(\frac{x-j}{m}\right)^{h n}
$$

Theorem 1. The collision probability of acBF is $\ll \frac{1}{2}$.

Proof. Let, $n$ be the number of unique entries and $m=n^{2}$ be the number of cells in the filter, then a $\left(\begin{array}{l}n \\ 2\end{array}\right)$ numbers of key pair may collide with collision probability of $\frac{1}{m}$. The total collision probability is [45]

$$
\begin{aligned}
& E[X]=\left(\begin{array}{l}
n \\
2
\end{array}\right) \times \frac{1}{m} \\
& =\left(\begin{array}{l}
n \\
2
\end{array}\right) \times \frac{1}{n^{2}}<\frac{1}{2}
\end{aligned}
$$

Equation (23) is with an assumption of a single modulus operator which is a prime number. However, the 2DBF uses three modulus operations, $3 \mathrm{DBF}$ uses four modulus operations, $4 \mathrm{DBF}$ uses five modulus operations, and 5DBF uses six 
modulus operations. All modulus operations are performed using prime numbers. The $\beta$ is the bits per cell. An item can consume at most one bit of a filter, however, an item can also consume less than a bit if and only if there is a collision. Therefore, rewriting the Equation (23),

$$
\begin{aligned}
E[X] & =\left(\begin{array}{l}
n \\
2
\end{array}\right) \times \frac{1}{\beta \times m} \\
E[X] & =\left(\begin{array}{l}
n \\
2
\end{array}\right) \times \frac{1}{\beta \times n^{2}} \\
E[X] & =\frac{n-1}{2 \times \beta \times n} \ll \frac{1}{2} \\
& \because \frac{n-1}{n}<1
\end{aligned}
$$

Therefore,

$$
E[X]<\frac{1}{2 \times \beta} \ll \frac{1}{2}
$$

In modern computers, $\beta$ is $64-$ bits, and thus, collision probability is $<0.007$. Therefore, the collision probability of acBF is $\ll \frac{1}{2}$.

Corollary 1. $2 D B F$ has higher collision probability than $3 D B F, 4 D B F$, and $5 D B F$. Similarly, $3 D B F$ has higher collision probability than $4 D B F$ and $5 D B F$, and so on.

Table II clearly depicts the probability of returning true positive, false positive or true negative by acBF. If $2 \mathrm{DBF}$ returns negatives in a membership query of an item, then it is guaranteed that the item does not exist. Otherwise, test the membership of the item in $2 \mathrm{DBF}^{\prime}$, and so on. $2 \mathrm{DBF}$ either returns true or false. Thus, the probability of returning false by $2 \mathrm{DBF}$ is $\frac{1}{3}$. Similarly, the probability of $2 \mathrm{DBF}^{\prime}$ is $\frac{1}{3}$, and so on.

Theorem 2. acBF is free from false negative.

Proof. acBF uses 2BDF, 3DBF, 4DBF, and 5DBF for higher accuracy. These variants of filters do not have a false negative. If a bit contains ' 1 ', then the filter returns true, and false otherwise. False negative occurs when the bits are reset in the deletion process. The bits share information with other items. Then, deletion causes removal of all items that shares the particular bit. Thus, if the deleted item shares information with other items, then there is a false negative. Because, bit is reset in the time of deletion of an item. On the contrary, the filters do not permit deletion. Hence, acBF inherits the properties of these filters, namely, $2 \mathrm{BDF}, 3 \mathrm{DBF}, 4 \mathrm{DBF}$, and 5DBF. Therefore, it is free from false negative.

Theorem 3. acBF achieves a guaranteed accuracy of $99.98 \%$.

Proof. Let, $P_{1}, P_{1}^{\prime}, P_{2}, P_{2}^{\prime}, P_{3}, P_{3}^{\prime}, P_{4}$, and $P_{4}^{\prime}$ be the FPP of $2 \mathrm{DBF}, 2 \mathrm{DBF}^{\prime}, 3 \mathrm{DBF}, 3 \mathrm{DBF}^{\prime}, 4 \mathrm{DBF}, 4 \mathrm{DBF}^{\prime}, 5 \mathrm{DBF}$, and $5 \mathrm{DBF}^{\prime}$ respectively. The FPP of $5 \mathrm{DBF}^{\prime}$ is the FPP of acBF. Therefore, the FPP of acBF is FPP of $5 \mathrm{DBF}^{\prime}$ of $5 \mathrm{DBF}$ of $4 \mathrm{DBF}^{\prime}$ of $4 \mathrm{DBF}$ of $3 \mathrm{DBF}^{\prime}$ of $3 \mathrm{DBF}$ of $2 \mathrm{DBF}^{\prime}$ of $2 \mathrm{DBF}$. Let us, test the membership of $\mathcal{Y}$ in acBF. First, test whether $\mathcal{Y} \in$ $2 D B F$ is true or not. If $\mathcal{Y} \notin 2 B D F$, then $\mathcal{Y}$ does not exist and no further examination is required. Otherwise, $\mathcal{Y}$ may exists. Hence, test the input item $\mathcal{Y}$ in $2 D B F^{\prime}$. If $\mathcal{Y}^{\prime} \notin 2 D B F^{\prime}$, then $\mathcal{Y}$ does not exist and concludes. Otherwise, check whether $\mathcal{Y} \in 3 D B F$ is true or not and so on. The FPP of $2 \mathrm{DBF}$ is $P_{1}$. We assume that at most $P_{1}$ is $\frac{1}{3}$, i.e., either $2 \mathrm{DBF}$ can return positive or negative. Similarly, the $P_{1}^{\prime}, P_{2}, P_{2}^{\prime}, P_{3}, P_{3}^{\prime}$, $P_{4}$, and $P_{4}^{\prime}$ also have the same probability with $P_{1}$. Therefore, the negative probability is

$$
\prod_{i=1}^{4} P_{i} \times P_{i}^{\prime}
$$

Therefore, the probability of the acBF returns positive is $\frac{1}{3^{8}}$ by the Equation (28). Assuming, all filters returned true. The "true" results of the filters can be true positive or false positive. There are $3^{8}$ possibilities of the returned result be false. Hence, the total false positive probability is $\frac{1}{3^{8}}=0.00015$. Therefore, the probability of true positive $1-\frac{1}{3^{8}}=0.9998$.

Corollary 2. The false positive probability of acBF is 0.00015 .

Theorem 4. Each item occupies at most 8-bits in acBF.

Proof. An item is inserted into eight filters in acBF, namely, $2 \mathrm{DBF}, 2 \mathrm{DBF}^{\prime}, 3 \mathrm{DBF}, 3 \mathrm{DBF}^{\prime}, 4 \mathrm{DBF}, 4 \mathrm{DBF}^{\prime}, 5 \mathrm{DBF}$, and $5 \mathrm{DBF}^{\prime}$. Each item consumes at most a single bit or less in a 2DBF, 3DBF, 4DBF, or 5DBF. An item can share a bit if there is a collision between two items. Thus, each item occupies at most eight bits in eight filters. However, if there is a collision, then an item occupies less than eight bits.

Theorem 5. The time complexity of insertion and testing membership of acBF is $O(1)$.

Proof. acBF uses an optimal time to insert and test. Let us assume, $\mathcal{C}$ be the mean time taken by Murmur hashing and $\mathcal{L}$ be the average string length. 2DBF invokes Murmur hashing for once, performs three modulus operations, and read the input string. Therefore, the time complexity is

$$
\mathcal{C}+\mathcal{L}+3
$$

Similarly, 3DBF uses same time amounts as shown in Equation (29) except $3 \mathrm{DBF}$ performs a modulus operation more than 2DBF. Hence, the time complexity of 3DBF is

$$
\mathcal{C}+\mathcal{L}+4
$$

and so on. Therefore, the total time complexity is

$$
\begin{gathered}
8 \times \mathcal{C}+8 \times \mathcal{L}+3+3+4+4+\ldots+6+6 \\
=8 \times \mathcal{C}+8 \times \mathcal{L}+36 \\
=O(\mathcal{C}+\mathcal{L})
\end{gathered}
$$

The $\mathcal{C}$ and $\times \mathcal{L}$ are constant. Hence, the total time complexity of an item is to insert or testing membership is $O(1)$. 
TABLE II

A TABLE OF TRUe POSITIVE (T), FALSE POSITIVE (FP) AND TRUE NEGATIVE (F) AT THE TIME OF QUERYING AN ITEM IN ACBF

\begin{tabular}{|c|c|c|c|c|c|c|c|c|}
\hline $2 \mathrm{DBF}$ & $2 \mathrm{DBF}^{\prime}$ & $3 \mathrm{DBF}$ & $3 \mathrm{DBF}^{\prime}$ & 4DBF & $4 \mathrm{DBF}^{\prime}$ & $5 \mathrm{DBF}$ & $5 \mathrm{DBF}^{\prime}$ & $\operatorname{Pr}$ \\
\hline $\mathrm{T} / \mathrm{F} / \mathrm{FP}$ & & & & & & & & $\frac{1}{3}$ \\
\hline $\mathrm{T}$ & $\mathrm{T} / \mathrm{F} / \mathrm{FP}$ & & & & & & & $\frac{1}{3}$ \\
\hline $\mathrm{T}$ & $\mathrm{T}$ & $\mathrm{T} / \mathrm{F} / \mathrm{FP}$ & & & & & & $\frac{1}{3}$ \\
\hline $\mathrm{T}$ & $\mathrm{T}$ & $\mathrm{T}$ & $\mathrm{T} / \mathrm{F} / \mathrm{FP}$ & & & & & $\frac{1}{3}$ \\
\hline $\mathrm{T}$ & $\mathrm{T}$ & $\mathrm{T}$ & $\mathrm{T}$ & $\mathrm{T} / \mathrm{F} / \mathrm{FP}$ & & & & $\frac{1}{3}$ \\
\hline $\mathrm{T}$ & $\mathrm{T}$ & $\mathrm{T}$ & $\mathrm{T}$ & $\mathrm{T}$ & $\mathrm{T} / \mathrm{F} / \mathrm{FP}$ & & & $\frac{1}{3}$ \\
\hline $\mathrm{T}$ & $\mathrm{T}$ & $\mathrm{T}$ & $\mathrm{T}$ & $\mathrm{T}$ & $\mathrm{T}$ & $\mathrm{T} / \mathrm{F} / \mathrm{FP}$ & & $\frac{1}{3}$ \\
\hline $\mathrm{T}$ & $\mathrm{T}$ & $\mathrm{T}$ & $\mathrm{T}$ & $\mathrm{T}$ & $\mathrm{T}$ & $\mathrm{T}$ & $\mathrm{T} / \mathrm{F} / \mathrm{FP}$ & $\frac{1}{3}$ \\
\hline
\end{tabular}

\section{DISCUSSION}

Many systems require high accuracy in Bloom Filter, including Computer Networking. Hence, acBF offers a guaranteed minimum accuracy of $99.98 \%$. A false positive is impractical with $m=n^{2}$ at load factor of $\alpha=\frac{n}{m}$. Besides, the key strength of the acBF is the maximum false positive probability of 0.00015. Also, acBF offers an impressive performance in a practical scenario. Because, bitwise operators are very fast. For lookup and insertion, eight locations are accessed in acBF. Moreover, the Murmur hashing greatly boosts up the performance of acBF. The acBF is constructed using multidimensional filters, but these filters are placed hierarchical fashion. This hierarchy consists of eight stages in acBF. acBF reduces the probability of false positive in each stage. On the contrary, the acBF inherits the drawbacks of $\mathrm{rDBF}$ [41]. The size of $\mathrm{rDBF}$ (2DBF, 3DBF, etc.) is fixed and it cannot be expanded once allocated. The dimensions of the rDBF define the size of the filters. The dimensions are prime numbers and these prime numbers greatly alleviate in distributing the input items in the filter.

\section{CONCLUSION}

In this paper, we have presented a novel Bloom Filter, called acBF. acBF offers a very high accuracy Bloom Filter with a guaranteed accuracy of $99.98 \%$. In addition, acBF also features an impressive FPP of 0.00015. Besides, acBF also uses optimal memory space per item, i.e., an item consumes at most 8 -bits irrespective of input item size. Also, acBF takes $O(1)$ time complexity to insert an item and test membership of an item. acBF deploys eight Bloom Filters to eliminate the false positive at eight stages. The false positive is very rare in acBF and it is almost impossible in a practical scenario. In addition, acBF does not have a false negative. However, the acBF depends on the prime numbers and the prime numbers define the size of the filter. Also, cell size $\beta$ must be a prime number or an odd number to avoid collision. For instance, the number 61 is a prime number for $\beta=64$. acBF also provides a collision probability of $\frac{1}{2 \times \beta}$ which is less than 0.007 for $64-$ bits assumption of per cell. Furthermore, acBF is independent from number of hash functions. Besides, acBF does not depend on the input item size. Moreover, acBF offers early detection of negative results. acBF uses static Bloom Filter which enhance the performance. Dynamic Bloom Filter sacrifices readjustment cost upon increasing the input size. However, a few Megabytes of acBF can scale billions of input items, since acBF is independent from the input item size. Undoubtedly, acBF can be deployed in many applications to boost up the system performance and accuracy, namely, Big Data, Network traffic, Network Security, Information Centric Network and other networking domain, IoT, Fog Computing, Bioinformatics, Biometrics, Databases, and Cloud Computing.

\section{REFERENCES}

[1] B. H. Bloom, "Space/time trade-o s in hash coding with allowable errors," Comm. of the ACM, vol. 13, no. 7, pp. 422-426, 1970.

[2] F. Chang, J. Dean, S. Ghemawat, W. C. Hsieh, D. A. Wallach, M. Burrows, T. Chandra, A. Fikes, and R. E. Gruber, "Bigtable: A distributed storage system for structured data," ACM Trans. Comput. Syst., vol. 26, no. 2, pp. 4:1-4:26, 2008.

[3] R. Patgiri, S. Nayak, and S. K. Borgohain, "Preventing ddos using bloom filter: A survey," EAI Endorsed Transactions on Scalable Information Systems: Online First, vol. 5, no. 19, pp. 1-9, 112018.

[4] S. Geravand and M. Ahmadi, "Bloom filter applications in network security: A state-of-the-art survey," Computer Networks, vol. 57, no. 18, pp. $4047-4064,2013$.

[5] A. Singh, S. Garg, S. Batra, N. Kumar, and J. J. Rodrigues, "Bloom filter based optimization scheme for massive data handling in iot environment," Future Generation Computer Systems, vol. 82, no. 2018, pp. 440-449, 2017.

[6] C. Rathgeb, F. Breitinger, C. Busch, and H. Baier, "On application of bloom filters to iris biometrics," IET Biometrics, vol. 3, no. 4, pp. 207218, 2014.

[7] S. Xiong, Y. Yao, S. Li, Q. Cao, T. He, H. Qi, L. Tolbert, and Y. Liu, "kBF: Towards approximate and bloom filter based key-value storage for cloud computing systems," IEEE Transactions on Cloud Computing, vol. 5, no. 1, pp. 85-98, 2017.

[8] P. Melsted and J. K. Pritchard, "Efficient counting of k-mers in dna sequences using a bloom filter," BMC Bioinformatics, vol. 12, no. 1, p. $333,2011$. 
[9] S. D. Jackman, B. P. Vandervalk, H. Mohamadi, J. Chu, S. Yeo, S. A. Hammond, G. Jahesh, H. Khan, L. Coombe, R. L. Warren et al., "ABySS 2.0: resource-efficient assembly of large genomes using a bloom filter," Genome research, vol. 27, no. 5, pp. 768-777, 2017.

[10] S. Y. Nam, H.-D. Kim, and H. S. Kim, "Detector sherlock: Enhancing trw with bloom filters under memory and performance constraints," Computer Networks, vol. 52, no. 8, pp. 1545 - 1566, 2008.

[11] P. Xiao, Z. Li, H. Qi, W. Qu, and H. Yu, "An efficient ddos detection with bloom filter in sdn," in 2016 IEEE Trustcom/BigDataSE/ISPA. Tianjin, China: IEEE, 2016, pp. 1-6.

[12] W. Liu, W. Qu, X. He, and Z. Liu, "Detecting superpoints through a reversible counting bloom filter," The Journal of Supercomputing, vol. 63, no. 1, pp. 218-234, Jan 2013.

[13] D. Guo, Y. He, and P. Yang, "Receiver-oriented design of bloom filters for data-centric routing," Computer Networks, vol. 54, no. 1, pp. 165 174, 2010.

[14] I. Nikolaevskiy, A. Lukyanenko, T. Polishchuk, V. Polishchuk, and A. Gurtov, "isbf: Scalable in-packet bloom filter based multicast," Computer Communications, vol. 70, no. Supplement C, pp. $79-85$, 2015.

[15] B. Ahlgren, C. Dannewitz, C. Imbrenda, D. Kutscher, and B. Ohlman, "A survey of information-centric networking," IEEE Communications Magazine, vol. 50, no. 7, pp. 26-36, July 2012.

[16] J. Lee, M. Shim, and H. Lim, "Name prefix matching using bloom filter pre-searching for content centric network," Journal of Network and Computer Applications, vol. 65, pp. 36 - 47, 2016.

[17] J. H. Mun and H. Lim, "New approach for efficient ip address lookup using a bloom filter in trie-based algorithms," IEEE Transactions on Computers, vol. 65, no. 5, pp. 1558-1565, 2016.

[18] W. Quan, C. Xu, J. Guan, H. Zhang, and L. A. Grieco, "Scalable name lookup with adaptive prefix bloom filter for named data networking," IEEE Communications Letters, vol. 18, no. 1, pp. 102-105, 2014.

[19] J. H. Mun and H. Lim, "Cache sharing using bloom filters in named data networking," Journal of Network and Computer Applications, vol. 90 pp. $74-82,2017$.

[20] W. Quan, C. Xu, J. Guan, H. Zhang, and L. A. Grieco, "Scalable name lookup with adaptive prefix bloom filter for named data networking," IEEE Communications Letters, vol. 18, no. 1, pp. 102-105, January 2014.

[21] Z. Li, K. Liu, Y. Zhao, and Y. Ma, "Mapit: An enhanced pending interest table for ndn with mapping bloom filter," IEEE Communications Letters, vol. 18, no. 11, pp. 1915-1918, Nov 2014.

[22] W. You, B. Mathieu, P. Truong, J. Peltier, and G. Simon, "Dipit: A distributed bloom-filter based pit table for ccn nodes," in 2012 21st International Conference on Computer Communications and Networks (ICCCN), July 2012, pp. 1-7.

[23] J. H. Mun and H. Lim, "Cache sharing using bloom filters in named data networking," Journal of Network and Computer Applications, vol. 90, pp. $74-82,2017$.

[24] E. Tong, W. Niu, G. Li, D. Tang, L. Chang, Z. Shi, and S. Ci, "Bloom filter-based workflow management to enable qos guarantee in wireless sensor networks," Journal of Network and Computer Applications, vol. 39, pp. $38-51,2014$.

[25] R. Patgiri, S. Nayak, and S. K. Borgohain, "Role of bloom filter in big data research: A survey," International Journal of Advanced Computer Science and Applications, vol. 9, no. 11, pp. 655-661, 2018.

[26] F. Putze, P. Sanders, and J. Singler, "Cache-, hash-, and space-efficient bloom filters," J. Exp. Algorithmics, vol. 14, pp. 4:4.4-4:4.18, 2010.

[27] B. Fan, D. G. Andersen, M. Kaminsky, and M. D. Mitzenmacher, "Cuckoo filter: Practically better than bloom," in Proceedings of the 10th ACM Intl. Conf. on Emerging Networking Experiments and Technologies, ser. CoNEXT '14. Sydney, Australia: IEEE, 2014, pp. 75-88.

[28] F. Bonomi, M. Mitzenmacher, R. Panigrahy, S. Singh, and G. Varghese, "An improved construction for counting bloom filters," in Proceedings of the 14th Conference on Annual European Symposium - Volume 14. London, UK, UK: Springer-Verlag, 2006, pp. 684-695.

[29] M. A. Bender, M. Farach-Colton, R. Johnson, R. Kraner, B. C. Kuszmaul, D. Medjedovic, P. Montes, P. Shetty, R. P. Spillane, and E. Zadok, "Don't thrash: How to cache your hash on flash," Proc. VLDB Endow., vol. 5, no. 11, pp. 1627-1637, Jul. 2012. [Online]. Available: http://dx.doi.org/10.14778/2350229.2350275

[30] P. S. Almeida, C. Baquero, N. Preguia, and D. Hutchison, "Scalable bloom filters," Information Processing Letters, vol. 101, no. 6, pp. 255 261, 2007.

[31] M. Naor and E. Yogev, "Tight bounds for sliding bloom filters," Algorithmica, vol. 73, no. 4, pp. 652-672, Dec 2015.

[32] G. Einziger and R. Friedman, "Tinyset- an access efficient self adjusting bloom filter construction," IEEE/ACM Transactions on Networking, vol. 25, no. 4, pp. 2295-2307, 2017.

[33] H. Lim, J. Lee, H. Byun, and C. Yim, "Ternary bloom filter replacing counting bloom filter," IEEE Communications Letters, vol. 21, no. 2, pp. 278-281, 2017.

[34] A. Crainiceanu and D. Lemire, "Bloofi: Multidimensional bloom filters," Information Systems, vol. 54, no. Supplement C, pp. 311 - 324, 2015.

[35] A. Craig, B. Nandy, I. Lambadaris, and P. Koutsakis, "Bloomflow: Openflow extensions for memory efficient, scalable multicast with multi-stage bloom filters," Computer Communications, vol. 110, no. Supplement C, pp. 83 - 102, 2017.

[36] D. Yang, D. Tian, J. Gong, S. Gao, T. Yang, and X. Li, "Difference bloom filter: A probabilistic structure for multi-set membership query," in 2017 IEEE International Conference on Communications (ICC). Paris, France: IEEE, 2017, pp. 1-6.

[37] D. C. Chang, C. Chen, and M. Thanavel, "Dynamic reordering bloom filter," in 2017 19th Asia-Pacific Network Operations and Management Symposium (APNOMS). Seoul, South Korea: IEEE, 2017, pp. 288-291.

[38] G. Lu, B. Debnath, and D. H. C. Du, "A forest-structured bloom filter with flash memory," in 2011 IEEE 27th Symposium on Mass Storage Systems and Technologies (MSST). Denver, CO, USA: IEEE, 2011, pp. $1-6$.

[39] G. Lu, Y. J. Nam, and D. H. C. Du, "Bloomstore: Bloom-filter based memory-efficient key-value store for indexing of data deduplication on flash," in 012 IEEE 28th Symposium on Mass Storage Systems and Technologies (MSST). San Diego, CA, USA: IEEE, 2012, pp. 1-11.

[40] B. Debnath, S. Sengupta, J. Li, D. J. Lilja, and D. H. C. Du, "Bloomflash: Bloom filter on flash-based storage," in 2011 31st International Conference on Distributed Computing Systems. Minneapolis, MN, USA: IEEE, 2011, pp. 635-644.

[41] R. Patgiri, S. Nayak, and S. K. Borgohain, "rDBF: A r-dimensional bloom filter for massive scale membership query," Journal of Network and Computer Applications, vol. 136, pp. 100-113, 2019.

[42] IOTTA SNIA, "Snia iotta repository," Retrieved on 15 Jan 2019 from http://iotta.snia.org/traces/, 2019.

[43] K. McKelvey and F. Menczer, "Design and prototyping of a social media observatory," in Proceedings of the 22nd international conference on World Wide Web companion, ser. WWW '13 Companion. Rio de Janeiro, Brazil: ACM, 2013, pp. 1351-1358. [Online]. Available: http://dl.acm.org/citation.cfm?id=2487788.2488174

[44] F. Grandi, "On the analysis of bloom filters," Information Processing Letters, vol. 129, no. Supplement C, pp. 35 - 39, 2018.

[45] T. H. Cormen, C. E. Leiserson, R. L. Rivest, and C. Stein, Introduction to algorithms. USA: MIT press, 2009. 